\title{
Introduction: Letter to the grammar writer
}

The SignGram Blueprint is a tool designed to guide language specialists and linguists as they write a reference grammar of a sign language. This tool consists of two main components: the Checklist and the Manual.

The Checklist contains a list of linguistic constructions and phenomena that a sign language grammar should contain. Thus, it can be considered as a suggestion for the table of contents of the reference grammar to be written.

The Manual, on the other hand, guides the grammar writer in four ways, by providing:

(i) basic, background information on the linguistic constructions and phenomena listed in the Checklist;

(ii) guidelines on how to identify and analyze these grammar points;

(iii) suggestions for data elicitation techniques and materials; and

(iv) relevant bibliographic information that the grammar writer can consult during his/her research.

The Manual also contains a separate sub-component, the Glossary, which provides the definitions of certain linguistic terms used in the Manual.

In the following, we describe in more detail how the grammar writer can use the components of the Blueprint. However, before we move on to that, we would like to explain the context in which the Blueprint has been created, the reasons that lead us to think it is needed, and the choices we have made while writing it. We start by briefly discussing what grammar writing involves and then continue with describing the structure of the Blueprint in more detail.

\section{Grammatical descriptions, why?}

Sign language research has advanced rapidly over the past few decades, but it still faces an important stumbling block: the grammatical descriptions available for specific sign languages are incomplete and of varying reliability. Complete, thorough descriptions of sign languages are lacking, and this obviously has negative consequences - not only for the linguist studying a certain phenomenon (lack of knowledge about a certain undescribed aspect of the grammar might lead to a wrong characterization of a different, but related aspect), but also for a whole range of professionals who must rely on a comprehensive description of the language, such as sign language teachers of deaf children, trainers of sign language interpreters, teachers of sign language as a second language, clinicians involved in diagnosing language impairment and language pathologies, and speech therapists assessing language competence. 
Writing a grammar may serve very different goals, but no matter what type of grammar is intended, the content should be as accurate and comprehensive as possible. The SignGram Blueprint is an attempt at helping the grammar writer achieve this goal. However, the form of the final grammar will, of course, depend directly on the goal that the grammar writer has set. A reference grammar of a language, which intends to be exhaustive, is a very different product, both in terms of depth and presentation, from a didactic grammar meant as a support for language learning. Therefore, the Blueprint must be considered as a tool that the grammar writer needs to adapt to his or her needs.

It should be kept in mind that the Blueprint can also be useful to describe partial aspects of grammar, for instance in graduate thesis projects, and thus does not need to be implemented in its entirety. Nevertheless, when a basic grammatical description of a language is lacking, it is sometimes hard to describe phenomena in isolation. Therefore, cooperative work should be encouraged to produce comprehensive grammatical descriptions of sign languages, which are very much needed.

\section{How to use the Blueprint}

As mentioned above, the Blueprint has two main components: the Manual and the Checklist. The Manual has seven parts. A part covering the Socio-historical background is followed by six parts corresponding to the major components of grammatical knowledge: Lexicon, Phonology, Morphology, Syntax, Semantics, and Pragmatics. Each part starts with an introductory chapter explaining the function of the linguistic component under investigation (e.g. Morphology), the organization of the part, and suggestions on how to use it.

Subsequent chapters and major sections within each part also contain introductory subsections providing background information including definitions, classifications, and suggestions on how to overcome the methodological and analytical challenges the grammar writer might face. The remaining subsections in each chapter contain guidelines for identification and analysis of the grammar points. These are often followed by a section on Elicitation Materials. This section contains methodology and material suggestions for data elicitation. Each chapter ends with a list of bibliographic references of the literature that addresses these grammar points - be it from a general perspective of for a specific sign language.

The aim of the Manual is to guide the grammar writer in providing the descriptions of the grammar points listed in the Checklist. To make this tool user-friendly, we have striven to maintain a one-to-one correspondence between (sub-)headings in the Checklist and (sub-)headings in the Manual. The grammar writer can read the Manual as if it were an independent book or she/he can click on a heading in the Checklist to access the relevant information in the Manual. To demonstrate how the Manual may provide guidelines for the identification of a specific construction or phenomenon, 
let us give an example. The Morphology Part of the Checklist contains the heading '2.1.2.1. Noun-verb pairs'. This corresponds to the heading '2.1.2.1. Noun-verb pairs' in the Morphology Part of the Manual. In this subsection of the Manual, it is explained that a 'noun-verb pairs' heading in a reference grammar might be useful, since a morphological process by which action verbs can be derived from object nouns (say the verb SIT from the noun CHAIR) is attested in many sign languages. Representative examples of this morphological process from actual sign languages are given, and tests that can be used to distinguish the noun from the related verb are suggested. Finally, this subsection of the Manual contains the most relevant bibliographical references that deal with this phenomenon.

The Checklist and the Manual are offered as a suggestion and as a guide, but of course, it is up to the grammar writer to decide whether the relevant subsection makes sense in the grammar of the sign language he or she is describing. For example, if the morphological process by which verbs are derived from nouns is absent in that sign language, this section might be safely skipped. But if the grammar writer aims at putting his or her grammatical description in a typological perspective, he or she might opt to refer to the absence of such a process by contraposition to the languages that are mentioned to have it in the Manual. When developing the actual grammar for a given sign language, the grammar writer might want to depart from the structure proposed in the Checklist for a variety of reasons, both practical and conceptual. In fact, at various points of the Manual explicit suggestions are made for an alternative organization of the grammar.

In general, we expect that while the most general headings should be relevant for all sign languages (say, '1.2. Interrogatives' in the Syntax Part of the Checklist and the Manual), more specific sub-headings might be relevant only for a subset of sign languages. For example, '1.2.3.6. Split between the wh-sign and its restriction' is needed only for those sign languages in which an interrogative sign corresponding to 'which' can be separated from its restriction, say a noun like 'book'.

Also, note that the different parts of the Checklist and the Manual such as Syntax and Morphology are internally structured with an independent numeration. We hope that the independence of each part will help the grammar writer who might be interested in describing just a single component, say only the morphology or the syntax of the sign language studied.

Since we hope the Blueprint will be used by a wide range of language specialists, we have made an effort to keep the language as accessible as possible, and have tried to avoid technical, linguistic jargon. We have worked under the assumption that the 'grammar writer', who is the main target user of the Blueprint, does not need to be a professional linguist, although we assume familiarity with basic linguistic notions and grammatical concepts specific to sign languages. We also assume that he or she is acquainted with one or more sign languages.

The Blueprint is a product of several authors. However, we made all possible efforts to harmonize the style. For example, a potential source of confusion can be 
generated by the use of the term 'word' or 'sign' for the lexical unit of a sign language. As a rule of thumb, we used the term 'sign' except for linear order facts and some prosodic or morphological descriptions where the terms 'prosodic word', 'word order', and 'word-internal' will be used.

The Blueprint helps the reader with linguistic terminology in two ways: one is the Glossary. A number of linguistic terms in each section is automatically linked to the Glossary. The full list of glossary entries can also be found at the end of the Manual.

The other helpful tool is the cross-referencing between sections and parts of the Manual by means of hyperlinking. Typically, if there is a term/concept used in a section where it is mentioned but not described, a hyperlink connects it to the section where it is explained. In other cases, the section where one set of properties (for instance, syntactic properties) of a phenomenon is discussed is linked to another section where another set of properties (for instance, prosodic properties) are addressed. This will equip the grammar writer with a wider background knowledge on the topic and enable him/her to approach it from more than one angle if she/he intends to do so.

We mentioned that, in most cases, there is a one-to-one correspondence between the Checklist and the Manual. However, there are cases in which this correspondence does not hold. These cases are due to the fact that the Checklist contains only the list of linguistic features that should be described in a grammar. Therefore, the sections of the Manual that are more methodological in nature (typically, the introductory sections in chapters and major sections devoted to definitions, methodological and analytical challenges, elicitation materials, and references) do not have a correspondence in the Checklist. However, these methodological sections are numbered in a special way, so that they do not obstruct the parallel structures of the Checklist and the Manual.

The second area in which the one-to-one correspondence does not hold is due to a basic choice we made when we decided on the general design of the Blueprint. We believe that traditional grammars, even the most complete reference grammars available for better-studied spoken languages, tend to neglect the dimension of meaning. It is instructive in this regard to notice that in the average descriptive grammar, no comprehensive section is devoted to semantics and pragmatics; rather, the discussion of meaning aspects is usually distributed across sections describing formal aspects such as lexicon, morphology, or syntax.

We think that these traditional choices do not reflect recent linguistic achievements about the semantics and pragmatics of natural languages (spoken or signed). In addition, the traditional structure typically leads to a blending of formal and functional categories in the grammatical descriptions. One typical example is temporal categories. In many languages, the (formally unmarked) verbal present tense form is not only used to refer to the present but also to refer the future (and sometimes even to the past). Therefore, the grammatical category of tense must not be conflated with the semantic notion of tense. For this reason, we have devoted an entire part of the Blueprint to the elucidation of concepts related to meaning. 
We present a couple of illustrative examples of why having fully developed Semantic and Pragmatics parts can be useful. The first still involves the 'tense' category. Some traditional grammars tend to conflate the discussion of tense and aspect, especially in languages in which the same morpheme express both a tense and an aspect specification. Unlike more traditional grammars, the Manual includes two sections in which these concepts are explained from a formal perspective and a meaning perspective. As the sections on tense and aspect are already present in the Morphology part (form) of the Checklist, in order to avoid a duplication, there is no Semantics part (meaning) in the Checklist, but the relevant semantic notions are displayed in the Semantics part of the Manual for the grammar writer as important background information for investigating their potential morphological realizations in the target language.

Similarly, a section called 'conditional clauses' is only present in the Syntax part of the Checklist describing possible formal aspects of such clauses. Nevertheless, the Manual contains a section in the Semantics part about the meaning of conditionals, since we think that a proper description of this construction cannot leave out the meaning dimension. However, other aspects of meaning, especially those related to pragmatic aspects of meaning such as discourse structure, figurative meaning, and communicative interaction, do have a counterpart in the Checklist, because it is justified to have them as free-standing sections in a descriptive grammar. Since all semantic concepts are also addressed from a formal perspective in the Lexicon, Morphology, and Syntax parts, the Checklist does not contain a part on Semantics. By contrast, the part on Pragmatics discusses aspects of meaning beyond the sentence level and is therefore included in the Checklist. With the general move to treat semantic and pragmatic aspects on an equal footing with other grammar components, we mean to boost description and analysis of semantic and pragmatic properties in signed languages, which have lagged behind until quite recently.

\section{Methodological choices}

We mentioned previously that we have adopted a plain, non-technical style, and that it is our hope that non-professional linguists will also be able to use the Blueprint. However, we must stress that this choice is not due to an anti-theoretical or anti-formalist attitude. On the contrary, the scientific directors of the Blueprint are all formal linguists who are convinced that no adequate empirical description is possible without the lens provided by modern linguistic theories. An a-theoretical description does not exist. What is considered a-theoretical is often a description that assumes commonsense, naïve conceptions, instead of more sophisticated notions from current linguistic theories that invariably help sharpen the empirical description. Therefore, the organization of the Checklist and the content of the Manual is implicitly theorydriven. Although the specific analyses that informed our choices are not at the center 
of the stage, they can be retrieved by looking at the references that close each chapter of the Manual. This sometimes has a relative influence on the terminological choices made here (for instance, the term 'agreement verb' is used), but alternative denominations existing in the literature are also mentioned ('directional' or 'indicating verbs' for the example at hand).

A question that naturally arises when one projects a skeleton for sign language grammars is to what extent this should be similar to a grammar for spoken languages. The issue is tricky, even more so because no comprehensive reference grammar for any sign language exists yet. We have started from the assumption that sign languages are the products of the same language faculty that gave rise to spoken languages. So in principle, the main analytical categories that have been elaborated in the linguistic research on spoken language (for example, phonological features, verbal inflection, subordination, or implicature) and that have been fruitfully applied in spoken language research should be useful categories for sign languages as well. Thus, in those cases in which there is no sufficient information on how sign languages express a certain grammatical concept or construction, we referred to the findings on typologically diverse spoken languages, keeping in mind that if a certain linguistic phenomenon or construction has been observed in a group of spoken languages, it has the potential to be observed in the sign language studied.

Such transfer from the generalizations on spoken languages is undoubtedly useful; however, it is not sufficient. It is also very well known that the visuo-spatial modality does shape the way language is expressed, and new, modality-specific categories should at times be employed to describe sign language phenomena (for example, non-manual marking, classifier predicates, and role-shift). It is an open question whether these categories are really unique to the signed modality or correspond to mechanisms that are present in spoken languages, albeit in a less prominent form, thus having led to their exclusion from spoken language grammars. These types of questions are very important, but the Blueprint is not the place to find answers to them, since our goal is to offer adequate descriptive tools rather than to investigate the underlying issues. Thorough descriptive work on many more sign languages will hopefully contribute to (partially) answering those questions at some point by relying on more solid empirical ground. A separate issue concerns iconicity. The fact that some signs incorporate iconic features has consequences for the structure of the grammar at all levels. However, the effects of iconicity are not the same in the lexicon and in syntax, for instance. Thus, rather than having an independent section on iconicity, we decided to discuss its effects whenever they are immediately relevant for a specific aspect of the grammar or a grammatical phenomenon.

At first sight, the Checklist may look superficially similar to the table of contents of a reference grammar of a spoken language. However, we would like to stress that a category identified in spoken language may involve different exponents and linguistic processes in sign language. The Manual contains multiple examples of this where such differences are highlighted and explained in detail. For example, while compound is a 
standard grammatical concept in morphology and is found in the Checklist, its application to sign languages raises some non-trivial questions. One is how to analyze compounds with multiple articulators that work in parallel and relatively independently from each other, for example, those in which one hand articulates (part of) one sign while the other one simultaneously articulates (part of) another sign.

As a final note on the Manual, we would like to point out that the current state of the art in sign language research has had some effect on the varying degree of detail across chapters and sections. Where necessary, we have tried to compensate for the existing gaps on the basis of the available linguistic information on spoken languages, as mentioned above. The grammar writer interested in further deepening his or her grammatical knowledge is encouraged to consult the selection of bibliographic pointers included at the ends of sections and chapters.

In some cases, original research has been conducted specifically for the preparation of the Blueprint, since the phenomenon to be described had not been explored at all for sign languages. In these cases, the original findings are the starting point for the relevant section. This is the case, for instance, in the section on imperatives in the Syntax part.

\section{The Blueprint and the SignGram COST Action}

The Blueprint is the main product of the SignGram COST Action (Action IS1006 "Unraveling the grammars of European sign languages: pathways to full citizenship of deaf signers and to the protection of their linguistic heritage", website: http://signgram. eu). COST is a European network of nationally funded research activities which aims to promote and finance cooperative scientific projects with a specific goal. The SignGram COST Action started in 2011 and ended in 2015; its main goal was the creation of the Blueprint. Researchers from 13 COST countries (Austria, Denmark, France, Germany, Greece, Iceland, Italy, Netherlands, Norway, Poland, Spain, Turkey, and the United Kingdom) and two COST International Partner Countries (Argentina and Australia) took part in the Action. COST funded the following scientific activities: the meetings in which the design of the Blueprint was discussed and decided, scientific missions between the partners, and summer schools for junior researchers who want to start working in the sign language field, as well as four editions of a conference that has become a major venue for sign language researchers (FEAST, Formal and Experimental Advances in Sign Language Theory). Another activity promoted by the SignGram Action is the creation of a repository of materials that have been used for the elicitation of signs or structures by researchers in Europe and beyond. The repository can be found at the following link:

https://corpus1.mpi.nl/ds/asv/;jsessionid=A0026AAA3C521F75EC5ADF8C93354297?0.

Finally, COST has made it possible for the Blueprint to be freely available to everyone as an open-access publication. 
It is important to highlight that the new research project SIGN-HUB (2016-2020) funded by the Horizon2020 program of the European Commission has as one of its goals to implement the Blueprint to write on-line grammars of the following sign languages: DGS, LIS, LSE, LSC, NGT, and TID. This will make it possible to have the grammatical descriptions directly online and available to everyone once they have been validated.

\section{The social dimension of the Blueprint}

When we started the SignGram COST Action, we were motivated by scientific questions, since we are linguists. However, as is often the case for linguists working on neglected and ostracized languages (and sign languages still belong to this category!), we also had in mind a social dimension. This is what we wrote in the application we submitted to COST in 2010:

\footnotetext{
"Despite significant advances, linguistic knowledge of languages in the visuo-gestural modality is still sketchy and incomplete. This becomes an unsurmountable handicap when inclusive educational policies are proposed, as no reliable grammatical descriptions are available that could constitute the appropriate basis for curriculum development and teaching materials in bilingual-bicultural programmes, sign language (SL) teaching or SL interpreter training. As a result, the responsibility of describing the basic aspects of SLs for educational practices has been frequently left in the hands of teachers of the deaf, language therapists or SL teachers and interpreter trainers, who understandably often lack the required background. Only the best possible education in their SL, though, does guarantee personal development and full exercise of civil, linguistic and ultimately human rights for deaf signing individuals. This action aims to provide scientifically reliable tools in order to meet the broader societal challenge of ensuring equal rights for deaf signers across Europe, as expressed in several international legal initiatives (cf. Resolutions of the European Parliament in 1988 and 1998, Motion of the Council of Europe for the protection of sign languages 2001, UN Convention on the Rights of Persons with Disabilities 2006)."
}

At the end of the Action, we did create what we think is a scientifically reliable tool for writing grammars of sign languages. It is offered as a contribution to all those interested in setting out to accomplish this task. We hope that even when a grammar writer disagrees with some of our choices, this will be because the approach that we have adopted has advanced the discussion on how to study, describe, and ultimately reinforce the status of sign languages. 